\title{
Síndrome de hiperestimulación ovárica: complicación iatrogénica asociada a técnicas de reproducción asistida
}

Ovarian hyperstimulation syndrome: iatrogenic complication associated with assisted reproductive techniques

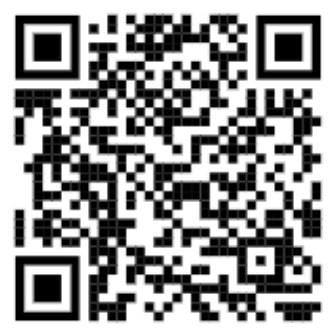

\author{
${ }^{1}$ Dra. Natalia Pérez Céspedes \\ Investigadora independiente, Alajuela, Costa Rica \\ (1) https://orcid.org/0000-0003-0132-9441 \\ ${ }^{2}$ Dra. Amaya Sainz Mejías \\ Investigadora independiente, Alajuela, Costa Rica \\ (D) https://orcid.org/0000-0003-0132-9441 \\ ${ }^{3}$ Dra. Ana Elena Camacho Solís \\ Investigadora independiente, San José, Costa Rica \\ (D) https://orcid.org/0000-0001-5276-1432
}

RECIBIDO

CORREGIDO

ACEPTADO

$09 / 04 / 2019$

$23 / 04 / 2019$

$30 / 04 / 2019$

\section{RESUMEN}

El síndrome de hiperestimulación ovárica es una de las complicaciones iatrogénicas más serias de las técnicas de reproducción asistida. Dicho síndrome está mediado por la liberación exagerada de factores vasoactivos por parte de las células de los folículos ováricos desarrollados mediante la estimulación ovárica controlada, y se caracteriza principalmente por aumento del tamaño ovárico junto con el desplazamiento de líquido y proteínas del espacio intravascular hacia un tercer espacio. Su diagnóstico es principalmente clínico apoyado en la ecografía

${ }^{1}$ Médico general, graduada de la Universidad de Costa Rica (UCR) Código médico: 15162. nataliaperezcespedes@gmail.com

2 Médico general, graduada de la Universidad de Costa Rica (UCR) Código sainzamaya@gmail.com

${ }^{3}$ Médico general, graduada de la Universidad de Costa Rica (UCR) Código médico: 15816. anaelena.cs25@gmail.com pélvica y su manejo depende de la clasificación según su severidad. Es posible identificar a las pacientes en riesgo de presentarlo y se deben aplicar estrategias preventivas previo, durante y posterior a la implementación de técnicas reproductivas asistidas para disminuir el riesgo de presentar esta complicación.

PALABRAS CLAVE: síndrome de hiperestimulación ovárica; técnicas reproductivas asistidas; medicina reproductiva; inducción de la ovulación; fertilización in vitro.

\section{ABSTRACT}

Ovarian hyperestimulation syndrome is one of the most serious iatrogenic complications of assisted reproductive techniques. This 
syndrome is mediated by the release of vasoactive factors from the cells of ovarian follicles developed through controlled ovarian stimulation, and it is mainly characterized by the increase in ovarian size along with a shift of fluid and proteins from the intravascular space into a third space. The diagnosis is mainly clinical, supported by pelvic ultrasound, and the management depends on its severity. It is possible to identify patients at risk, and preventive strategies should be applied prior, during and after the implementation of assisted reproductive techniques to lower the risk of developing this complication.

KEYWORDS: ovarian hyperestimulation syndrome; assisted reproductive techniques; reproductive medicine; ovulation induction; fertilization in vitro.

\section{INTRODUCCIÓN}

El síndrome de hiperestimulación ovárica (SHO) es una complicación iatrogénica potencialmente letal asociada a la estimulación ovárica realizada durante algunas técnicas de reproducción asistida (TRA) (1). Este síndrome tradicionalmente se caracteriza por la presencia de aumento en el volumen ovárico, ascitis, hemoconcentración, hipercoagulabilidad y trastornos hidroelectrolíticos que ocurren como consecuencia de una estimulación ovárica farmacológica (2). Sin embargo, aunque clásicamente se considera como una entidad de origen iatrogénico, también puede ocurrir de forma espontánea o asociado a condiciones no relacionadas con tratamientos de fertilidad; al respecto se describe en la literatura casos producidos por adenomas pituitarios, hipotiroidismo, neoplasias trofoblásticas secretoras de gonadotropina coriónica humana (hCG), tumores neuroendocrinos secretores de hormona folículo estimulante (FSH) 0 asociados al embarazo (3).
La incidencia de este cuadro varía según el contexto clínico, ya que el SHO clínicamente significativo afecta a un 2$3 \%$ de las pacientes que se someten a estos tratamientos, mientras que las formas leves ocurren en hasta un 20$30 \%$ de los ciclos de fertilización in vitro (FIV) (4). A pesar de ser una complicación poco común, cuando se presenta como SHO severo asocia alta morbilidad y mortalidad si no es manejado adecuadamente (5). Adicionalmente, con el aumento del uso de TRA para el manejo de la subfertilidad, se ha dado un aumento concomitante en los casos reportados, y por lo tanto la relevancia de este síndrome como entidad clínica (6).

El presente artículo constituye una revisión bibliográfica cuyo objetivo principal es permitirle al clínico identificar a las pacientes en riesgo e incluir al $\mathrm{SHO}$ dentro del diagnóstico diferencial, así como establecer una guía del manejo adecuado de las implicaciones clínicas asociadas. 


\section{FISIOPATOLOGÍA}

En la fisiología normal de la ovulación espontánea, los ciclos están usualmente asociados con el desarrollo de un único folículo, como resultado de retrocontrol negativo mediante el eje hipotálamohipófisis-ovario. Las células de la granulosa dentro de este folículo contienen péptidos vasoactivos y en el momento de la ovulación, la liberación de estas sustancias promueve cambios de neovascularización en la zona circundante a este. Sin embargo, en los tratamientos de reproducción asistida, se utilizan gonadotropinas exógenas que evitan los mecanismos de retrocontrol fisiológicos, y permiten el reclutamiento y desarrollo simultáneo de múltiples folículos ováricos (7). Posteriormente, se utiliza la administración de hCG exógena para simular el pico endógeno de la hormona luteinizante (LH), lo cual es estímulo para la maduración final de los ovocitos contenidos dentro de los folículos (8). A pesar de que ambas sustancias activan el mismo receptor, existen diferencias entre la LH y la hCG que causan una respuesta de distinta magnitud. La hCG presenta una vida media de 24 horas, mientras que la vida media de la LH es de menos de 60 minutos. Por lo tanto, la vida media más prolongada de la hCG causa una actividad luteotrópica mayor que la que ocurre en condiciones fisiológicas (9).

En la actualidad, la mayoría de los mecanismos fisiopatológicos que conducen a la aparición de este síndrome han sido identificados, aunque prosiguen los estudios para esclarecer los procesos involucrados en su surgimiento. Al respecto, se ha establecido que la administración de FSH y LH exógena, seguido de hCG provoca un fenómeno de hiperestimulación ovárica que en algunos casos se asocia al SHO (3). La administración de hCG en el contexto de TRA, provoca la luteinización folicular y la liberación acumulativa de los factores vasoactivos por parte de las células de la granulosa en los múltiples folículos desarrollados (7). Si estos factores son producidos de manera excesiva, pueden ocasionar el desplazamiento de líquido y proteínas del espacio intravascular a un tercer espacio (5), dicha pérdida de líquido hacia otros hacia otros compartimientos está asociado con las complicaciones más graves de este síndrome (3). Dentro de los factores vasoactivos responsables se incluyen factor de crecimiento vascular endotelial (VEGF), angiotensina II, interleucina 6 y factor de crecimiento similar a la insulina (5). Se ha propuesto que el VEGF presenta un papel importante en mediar el aumento de permeabilidad vascular en esta enfermedad, ya que se ha demostrado que la administración de hCG aumenta su expresión y que sus niveles séricos han demostrado correlación con la severidad del síndrome (1). Dentro de sus funciones se destaca el estímulo de procesos como el crecimiento folicular, la función del cuerpo lúteo, angiogénesis y estimulación vascular endotelial (2).

\section{PRESENTACIÓN CLÍNICA}

Se han descrito dos tipos de SHO según su momento de inicio. El SHO de inicio temprano aparece dentro de los primeros 10 días después de la administración de hCG. Esta presentación clínica está 
asociada con la respuesta ovárica exagerada a la administración de hCG exógena, teniendo además un carácter usualmente autolimitado en las pacientes que no conciben, y que típicamente resuelve al momento de la siguiente menstruación $(1,4)$. La presentación de inicio tardío aparece después de 10 días de la administración de la hCG exógena (1), estos casos se presentan cuando las mujeres conciben y se da una producción de hCG endógena asociada al embarazo temprano (9). Los casos que comprenden un $\mathrm{SHO}$ de inicio temprano seguido de SHO de inicio tardío usualmente resultan en formas más serias y prolongadas del síndrome (1).

Es crucial clasificar el caso según la severidad de los signos y síntomas que presente el paciente, ya que según el grado de severidad así será su manejo. Navot y colaboradores propusieron una clasificación práctica que aún se encuentra vigente $y$ es ampliamente utilizada (10). Se considera un caso leve ante la presencia de distensión y dolor abdominal leve, náuseas, vómitos, diarrea, una ecografía que indique ovarios aumentados de tamaño (usualmente menor a $8 \mathrm{~cm}$ ) y que no existan hallazgos significativos en los exámenes de laboratorio $(6,11)$. Si además de presentar las características del caso leve, el dolor abdominal aumenta y la ecografía muestra ovarios aumentados de tamaño entre 8 y $12 \mathrm{~cm}$ y la presencia de ascitis, se considera un caso moderado $(6,11)$.

Se clasifica como caso severo cuando la paciente presenta ascitis evidenciada por clínica $y / 0$ hidrotórax, ovarios aumentados de tamaño, disnea severa, oliguria, pruebas de función renal o hepáticas alteradas, hipoproteinemia, hematocrito mayor a $45 \%$ y/o leucocitos más de 15000/mL $(6,11)$. La clasificación más extrema, el grado crítico, ocurre cuando se presenta cualquiera de las siguientes características: ascitis a tensión asociada o no a derrame pleural o pericárdico, oliguria/anuria, fenómenos tromboembólicos, síndrome de distrés respiratorio severo y deterioro de los resultados de laboratorio: hematocrito mayor a 55\%, leucocitos más de 25000/mL (11,12).

El aumento de permeabilidad capilar mediado por factores humorales y la consecuente formación de un tercer espacio son las características principales del síndrome (4). Los síntomas pueden aparecer tan temprano como en las primeras 24 horas posteriores a la administración de hCG y pueden incrementar en severidad en los siguientes 7 a 10 días. Su presentación inicial puede cursar con distensión abdominal, causada por el aumento en el tamaño de los ovarios (1), posteriormente ocurre un desplazamiento de líquido y albúmina principalmente hacia la cavidad peritoneal, causando un aumento progresivo de la circunferencia abdominal.

Otras manifestaciones clínicas de la formación de un tercer espacio son edema en extremidades inferiores y acúmulo de fluido en las cavidades pleurales. Adicionalmente, la acumulación progresiva de líquido intersticial puede causar alteraciones respiratorias, diarrea y náuseas asociadas a edema intestinal, y alteración en el perfil hepático asociado al daño causado por edema a este nivel (3). El aumento en la severidad es el resultado de un aumento en la permeabilidad vascular, la pérdida de 
volumen intravascular y agravamiento de la ascitis, resultando en hipovolemia y hemoconcentración (8). Esta disminución del volumen a causa del desplazamiento de líquido a un tercer espacio puede contribuir a oliguria progresiva, inestabilidad hemodinámica y trastornos hidroelectrolíticos como hiponatremia e hiperpotasemia (3).

\section{DIAGNÓSTICO}

Para que exista la sospecha de este síndrome es indispensable la historia reciente de estimulación ovárica seguida de la ovulación o la administración de hCG (11). El diagnóstico es clínico y una vez identificado el síndrome, debe clasificarse como leve, moderado, severo o crítico (8). Toda paciente sintomática debe ser interrogada acerca de la duración de sus síntomas, su frecuencia y volumen urinario y si ha presentado aumento brusco de peso. Dentro de los diagnósticos diferenciales a descartar se encuentran la enfermedad pélvica inflamatoria, apendicitis, embarazo ectópico, hemorragias intraabdominales o torsión ovárica $(11,12)$.

La clasificación de la severidad toma en cuenta hallazgos en historia clínica, examen físico y ecografía. Se debe tomar en cuenta la edad de la paciente, la duración del cuadro, la cantidad y tamaño de los folículos durante la estimulación, así como análisis de sangre y ecografía pélvica (11). Los análisis iniciales deben incluir hemograma, electrolitos y examen general de orina para valorar el estado de hidratación de la paciente. También se realizan pruebas de función renal y hepática para valorar daño a órgano blanco (12).

El examen físico completo incluye la medición de sus signos vitales, peso corporal, circunferencia abdominal, explorar en busca de datos de derrame pleural y valoración de miembros inferiores en busca de signos de trombosis venosa profunda (12). El objetivo de realizar la ecografía pélvica es evaluar la cantidad y tamaño de los quistes luteales, el grado de ascitis y el aumento de tamaño de los ovarios, el cual siempre será bilateral. Al inicio del cuadro el aumento del tamaño ovárico puede enmascarar el líquido libre de la pelvis, por lo que puede pasar desapercibido en una ecografía transabdominal, es por esto que en los casos en los que la ascitis no es tan evidente se recomienda realizar ecografía transvaginal (13).

\section{MANEJO}

El manejo de este síndrome es principalmente de soporte porque suele ser autolimitado, sin embargo, dependerá de la gravedad con la que se presenten los síntomas y de si la paciente se encuentra embarazada en el momento de la presentación (12). Los casos clasificados como leves o moderados pueden ser tratados de forma ambulatoria y existe la posibilidad de regresión espontánea en los 10-14 días posteriores al inicio de la clínica, principalmente en los casos en los que no haya ocurrido la implantación (14). Por lo contrario, las pacientes que presenten casos severos o críticos deberán ser hospitalizadas para 
disminuir el riesgo de presentar complicaciones serias (8).

El manejo ambulatorio requiere un asesoramiento adecuado de la paciente, se le debe enfatizar la importancia de la ingesta de líquidos, con la recomendación de al menos 1 litro diario, e informar sobre los síntomas y signos de alarma. Dichos signos de alarma incluyen la presencia de disnea, disminución del volumen urinario, edemas en miembros inferiores 0 alteraciones neurológicas (12). Es recomendable un seguimiento continuo, al menos dos consultas por semana para evaluar laboratorios: hemograma completo, pruebas de coagulación, función hepática, función renal, electrolitos y ecografía pélvica, además de instruirlas para que registren su peso diariamente (14).

Con respecto a las pacientes que ameriten manejo intrahospitalario, estas requerirán mayor observación de sus signos vitales, un registro diario de la ingesta de líquidos, diuresis, circunferencia abdominal y peso, así como exámenes de laboratorio frecuentes (8). Se debe evaluar la ascitis y el tamaño ovárico por medio de ecografía y en caso de presentar datos de derrame pleural o pericárdico, realizar radiografía de tórax (14).

La clave del tratamiento consiste en mantener un adecuado estado de hidratación y equilibrio electrolítico, por lo tanto, se debe considerar la administración de fluidos intravenosos en caso de deshidratación o hemoconcentración (8). Se recomienda iniciar con soluciones cristaloides, una opción es iniciar con 1 bolo rápido de $1 \mathrm{~L}$ de solución salina al $0,9 \%$, seguido de solución de dextrosa al 5\%, de 125 a
$150 \mathrm{~mL} / \mathrm{h}$. El objetivo es mantener un gasto urinario mayor a $20-30 \mathrm{~mL} / \mathrm{h}$ y corregir la hemoconcentración $(12,14)$. Si con esta solución no se logra el objetivo, se recomienda cambiar a una solución coloide alternativa 0 expansores de plasma (12). El uso de expansores del plasma, como albúmina, manitol o plasma fresco congelado puede tener un efecto beneficioso al aumentar la presión oncótica para mantener el volumen intravascular, sin embargo deben utilizarse con precaución debido al riesgo de exacerbar la ascitis(14). La albúmina al $25 \%$ es el expansor de plasma más utilizado, en una dosis de $50 \mathrm{~g}$ infundida durante 4 horas (12). No se recomienda el uso de diuréticos de rutina, éstos pueden disminuir el volumen intravascular, causar hipotensión y aumentar el riesgo de trombosis venosa al aumentar la viscosidad sanguínea(14). Sin embargo, si se considera necesario su uso, pueden indicarse junto con solución coloide una vez que el volumen intravascular sea adecuado y se haya revertido la hemoconcentración (12).

La hemoconcentración, el estado de hipercoagulabilidad en el caso de las pacientes embarazadas y la inmovilización producto del internamiento hospitalario, aumentan el riesgo de presentar un tromboembolismo, por lo tanto, las pacientes requieren anticoagulación profiláctica con heparina de bajo peso molecular, como enoxaparina 40-80 mg/día, sin necesidad de monitoreo $(8,14)$. La duración de la anticoagulación depende de cada caso, sin embargo en pacientes embarazadas se recomienda continuarla al menos hasta el final del primer trimestre (4). A todas las pacientes se les debe informar que el reposo prolongado en cama 
aumenta el riesgo de trombosis y por lo tanto debe ser evitado, incluso en las pacientes hospitalizadas. La tromboprofilaxis también incluye el uso de medias compresivas o dispositivos de compresión neumática intermitente (12). Independientemente de la severidad del cuadro es indispensable el adecuado manejo del dolor, siendo el paracetamol y/o analgésicos opioides la primera línea de tratamiento. En todo caso es recomendable evitar los antiinflamatorios no esteroideos por su compromiso en la función renal y sus propiedades antiplaquetarias, que pueden interferir con el proceso de implantación $(8,12)$. Las náuseas y vómitos deben ser manejados con antieméticos considerados seguros en embarazo (12). Si la paciente presenta ascitis a tensión, que le provoca dolor abdominal, dificultad respiratoria o presenta oliguria, está indicado realizar paracentesis con el fin de evitar la progresión a casos más críticos (4). Incluso se ha observado que manejar a las pacientes de forma ambulatoria, programando paracentesis y rehidratación intravenosa cada 1-3 días es una alternativa segura para evitar la hospitalización (2). En casos como éstos, donde se requieran procedimientos invasivos, como la paracentesis, culdocentesis, cateterismos frecuentes o drenaje pleural, está indicada la profilaxis con antibióticos (14). Es importante destacar que la paracentesis debe realizarse siempre guiada por ecografía porque se han reportado casos de lesión ovárica y lesión vascular en paracentesis realizadas sin guía ecográfica (2).

Las pacientes clasificadas como grado crítico 0 que hayan presentado complicaciones, por ejemplo, tromboembolismo pulmonar, hidrotórax masivo, distrés respiratorio agudo, entre otras, requerirán internamiento en la unidad de cuidados intensivos y la terminación del embarazo se tomará en cuenta en el caso extremo en el que la vida de la paciente esté en riesgo (12).

\section{COMPLICACIONES}

Los eventos trombóticoscorresponden a la complicación del SHO que más amenaza la vida de estas pacientes (14). El riesgo de estos eventos aumenta asociado tanto a la hemoconcentración, como a otros factores como lahiperestrogenemia, antecedentes familiares y personales de trastornos de la coagulación, y el estado de hipercoagulabilidad que existe durante el embarazo. Un $75 \%$ de los casos de eventos trombóticos son de origen venoso, mientras que sólo un $25 \%$ son de origen arterial. Dentro de los casos asociados a tromboembolismo venoso, se destaca al tromboembolismo pulmonar como la consecuencia más grave. Otras complicaciones que pueden presentarse son el fallo renal y la insuficiencia respiratoria (15). Adicionalmente, se puede presentar hidrotórax, especialmente en hemitórax derecho, como cuadro de instauración aguda asociado a ascitis severa, por la transferencia de fluído abdominal al pecho. Esta complicación puede ser manejada mediante paracentesis. Sin embargo, en hidrotórax bilaterales 0 aquellos que persisten con derrame pleural posterior a la paracentesis, se prefiere el manejo mediante toracocentesis (14). 
Por otra parte, el aumento en el volumen ovárico secundario a la estimulación hormonal puede a su vez generar complicaciones como torsión y ruptura ovárica (16). El diagnóstico de estas entidades resulta particularmente difícil, ya que los síntomas son inespecíficos y se traslapan con los hallazgos del SHO, pero el dolor abdominal súbito, de inicio nocturno y que persiste por más de 24 horas sugiere compromiso anexial. El reconocimiento temprano de estas complicaciones es crucial para evitar la necrosis ovárica y preservar la fertilidad, y aunque la evaluación por ecografía puede ser de utilidad, no es una prueba específica 0 sensible para torsión ovárica. Por ello, la exploración quirúrgica es la primera línea para el diagnóstico y tratamiento de estas condiciones (17).

\section{PREVENCIÓN}

El SHO teóricamente puede ocurrir en cualquier mujer que se someta a estimulación ovárica controlada, pero hay ciertos factores, tanto primarios como secundarios, que aumentan el riesgo de presentar SHO clínicamente significativo. La identificación de dichos factores en las pacientes que se someterán a TRA es esencial para disminuir la incidencia de este síndrome y para establecer regímenes de prevención primaria y secundaria (2). Sin embargo, actualmente no se han descrito intervenciones que permitan eliminar por completo este riesgo (15).

\section{Factores de riesgos}

Primarios. La edad menor de 30 años es un factor de riesgo primario, esto porque se asocia a mayor reserva ovárica y de densidad de receptores de FHS $(7,15)$. De igual manera, un índice de masa corporal bajo se asocia a riesgo de $\mathrm{SHO}$ $(15,18)$. Otra población en riesgo son las pacientes con síndrome de ovario poliquístico (SOP), lo cual es importante de considerar debido a que este es el desorden endocrino más común en mujeres de edad reproductiva. En estas pacientes el riesgo aumentado se debe a la mayor sensibilidad y reacción exagerada ante las gonadotropinas que presentan estas pacientes (19). Adicionalmente, el antecedente de un episodio previo predispone a la recurrencia del mismo (15).

La hormona antimülleriana $(\mathrm{AMH})$ se perfila como un predictor muy promisorio de la respuesta ovárica. Una concentración previa al ciclo de estimulación de $\mathrm{AMH}$ mayor a 0,47 $\mathrm{pmol} / \mathrm{L}$ (3.36 $\mathrm{ng} / \mathrm{mL}$ ) predice el desarrollo de $\mathrm{SHO}$ con una sensibilidad de $90,5 \%$ y una especificidad de 81,3\% $(15,18)$. Inclusive estudios retrospectivos han documentado que una concentración de $\mathrm{AMH} \leq$ menor o igual a $0.18 \mathrm{pmol} / \mathrm{L}$ (1.26 $\mathrm{ng} / \mathrm{mL}$ ) puede identificar a mujeres que responderán al tratamiento de manera normal con una tasa de éxito del $98 \%$. Caso contrario con las concentraciones de estradiol sérico (E2) las cuales se han desempeñado con escasa evidencia para identificar mujeres en riesgo (18). De forma similar, dos estudios valoraron el valor predictivo de las concentraciones de inhibina $A$ y $B$ sin lograrse establecer una correlación entre ambos parámetros (2). Por otra parte, los marcadores ultrasonográficos, como el recuento de folículos antrales, predicen un riesgo de SHO moderado a severo cuando existe un conteo mayor o igual a 24, esto al compararlo con un conteo menor $(15,18)$. 
Secundarios. El monitoreo del estradiol sérico posterior a la estimulación ovárica constituye un importante predictor de riesgo, especialmente si se presenta un rápido aumento en su concentración o si su concentración es mayor a $2500 \mathrm{pg} / \mathrm{mL}$ $(15,18)$. De igual manera, el documentar más de 14 folículos en desarrollo de un diámetro superior a $11 \mathrm{~mm}$ el día de la administración de hCG predice riesgo de $\mathrm{SHO}$. Otros factores de riesgo secundarios son más de 20 ovocitos recuperados por punción ovárica, la elevación del factor de crecimiento endotelial vascular el día de la administración de hCG y un hematocrito mayor a $35 \%$ el día que se realice la punción folicular $(14,15)$.

A continuación se detallan recomendaciones para personalinzar los regímenes de tratamiento con el fin de disminuir el riesgo en pacientes con factores de riesgo primarios así como aquellas medidas recomendadas para utilizar en casos de pacientes que ya se hayan sometido a estimulación ovárica y cursen con una respuesta exagerada, con el objetivo de disminuir el riesgo de progresión a SHO $(14,15)$.

\section{Prevención primaria}

Este conglomerado de estrategias tiene como objetivo disminuir e inclusive evitar las posibilidades de que una mujer con factores de riesgos primarios llegue a desarrollar SHO (15). No se debe obviar que inclusive en mujeres sin ningún factor de riesgo se puede generar este síndrome $(14,18)$. Los factores de riesgos primarios, a diferencia de los secundarios, son aquellos presentes previos a un ciclo de estimulación (15). Por lo tanto, constituyen parte esencial de la valoración por parte del profesional en medicina previo al procedimiento. Los factores de riesgos secundarios evalúan ciertos parámetros de la respuesta ovárica relacionados al ciclo de estimulación con el objetivo de predecir el riesgo a desarrollar SHO (18).

1. Regímenes de inducción de la ovulación: Considerando a la subpoblación de mujeres con SOP y su riesgo aumentado de desarrollar $\mathrm{SHO}$, una terapia dirigida a la ovulación unifolicular sería la deseada (18). Para esto se puede considerar una reducción en la dosis utilizada de gonadotropinas, así como una disminución a la exposición de estas hormonas (14,15). Dosis menores de gonadotropinas evitan una respuesta ovárica exagerada y la cancelación del ciclo (15). Por lo tanto, se recomienda el uso de una terapia escalonada que inicia con dosis bajas de FSH (75 IU), las cuales aumentarían después de 14 días si se nota una respuesta ovárica caracterizada por la presencia de un folículo en desarrollo mayor a $10 \mathrm{~mm}$. Dicha dosis apropiado se continuará hasta obtener un folículo mayor o igual a $18 \mathrm{~mm}(14,18)$.

\section{Reducción del tiempo de exposición} a las gonadotropinas: Esta medida se logra a través de protocolos de estimulación en los cuales se retrasa la administración de la FSH hasta la fase folicular media o bien tardía (15).

3. Utilización de protocolos de estimulación con antagonistas de la hormona liberadora de gonadotropina (GnRH): Existe evidencia que respalda su uso ya que se asocia a una reducción del estradiol circulante y se requieren menores dosis de FSH. Asimismo, se ha 
observado que se mantienen tasas de gestación aceptables $(2,15)$.

4. Metformina como tratamiento coadyuvante: Especialmente en pacientes con SOP, reduce el riesgo de $\mathrm{SHO}$ alrededor de un $63 \%$ y aumenta las tasas de embarazo sin efectos en las tasas de natalidad $(14,15)$. Esta biguanida se asocia a la inhibición de la secreción de moléculas como VEGF, modulando la permeabilidad vascular (18). También disminuye la sensibilidad ovárica al FSH, mejorando la respuesta ante el ciclo de estimulación ovárica (20). Se recomienda iniciar dos meses previo al ciclo de estimulación una dosis diaria de 1000 a 2000 mg (14).

5. Individualización de los regímenes de FIV: Se recomienda aplicarun algoritmo que considere ciertos parámetros como el recuento de folículos antrales y la $\mathrm{AMH}$, como estrategia para predecir la posibilidad de una respuesta excesiva durante la estimulación ovárica e individualizar los regímenes aplicados $(14,18)$.

6. Alternativas para inducir la ovulación: La hCG exógena es el fármaco más comúnmente utilizando para simular el pico endógeno de $\mathrm{LH}$, pero su vida media prolongada eleva el riesgo de presentarse esta patología (14). A pesar de que la reducción de las dosis de hCG no ha demostrado mejoría en resultados clínicos, se ha planteado el uso de otros fármacos como alternativas para inducir la ovulación, basando esta decisión en los factores de riesgo que presente cada paciente. Sin embargo, no ha sido demostrada la capacidad de ningún agente en particular, que permita por si mismo eliminar por completo el riesgo de aparición de esta entidad $(15,18)$.

a) Los agonistas de $\mathrm{GnRH}$ desencadenan una elevación moderada y breve de gonadotropinas mediante la estimulación hipofisiaria, con acción por únicamente 24 a 36 horas $(15,18)$. El mismo es suficiente para inducir la ovulación y se puede considerar su uso en mujeres con alto riesgo $(2,16)$.

b) La LH recombinante simula un pico endógeno de $\mathrm{LH}$, y posee una vida media de 10 horas, por lo que podría asociarse a una disminución en la incidencia en pacientes de alto riesgo (18). Sin embargo, algunos estudios han documentado menores tasas de embarazo y una pobre relación costo beneficio $(14,18)$.

\section{Prevención secundaria}

1. Suspensión de la administración de hCG: Esta estrategia se basa en evitar la administración de hCG cuando se alcanza un nivel crítico de E2 (inferior a 3 $500 \mathrm{pg} / \mathrm{ml}$ ) o bien cuando se alcanza un número crítico de folículos en crecimiento (15). Una vez que los niveles de E2 han disminuido significativamente o alcanzan una meseta, se procede a la administración de hCG $(14,18)$. Esta suspensión puede realizarse por un máximo de tres días con el fin de no interferir con los resultados finales del ciclo (15).

\section{Criopreservacióndeovocitosoembrio}

nes: Con el fin de evitar una hiperestimulación tardía por el aumento de hCG proveniente del trofoblasto, se realiza una criopreservación de ovocitos o embriones. La transferencia posterior debe ser realizada en un ciclo en el cual 
la respuesta ovárica ante la hCG se haya normalizado $(15,18)$. Existe evidencia que respalda que esta técnica previene el SHO, sin embargo, no la suficiente para respaldarla como una técnica de rutina $(2,18)$.

3. Cancelación del ciclo: Esta medida drástica, pero definitiva para prevenir $\mathrm{SHO}$, consiste en cancelar el ciclo y evitar la administración de hCG cuando las ecografías muestren gran cantidad de folículos asociados altos niveles de estrógenos $(14,18)$. Sin embargo, puede implicar un alto impacto económico y psicológico en las pacientes que no debe obviarse (15).

4. Uso de agonistas de dopamina: Se ha planteado el uso de agonistas de la dopamina, como la cabergolina, con el fin de restringir el incremento de la permeabilidad vascular a causa del VEGF. Dicha intervención reduce la incidencia del SHO moderado, sin afectar la tasa de fecundación o implantación. La dosis recomendada es $0,5 \mathrm{mg}$ iniciando el día de la administración de hCG y continuándose por 8 días más $(14,15)$.

5. Aspirina: Un ensayo clínico aleatorizado comparó este fármaco con un grupo control, y demostró que la aspirina reduce la incidencia de $\mathrm{SHO}(2)$. A partir de esta evidencia, se ha planteado el uso de $100 \mathrm{mg}$ diarios de aspirina desde el primer día del ciclo de estimulación ovárico (14). Adicionalmente, este medicamento no afecta las tasas de embarazo (20).

\section{Otras medidas de prevención en estudio}

Se han propuesto otras medidas de prevención, como el uso de soluciones coloides para la inactivación de mediadores vasoactivos, pero estos no cuentan con suficiente evidencia que respalde su uso rutinario $(15,18)$. De igual forma, se ha planteado el uso de relcovaptan, un antagonista del receptor V1 de vasopresina, por su efecto inhibidor del VEGF pero se requiere aún de estudios complementarios. Por otra parte, la administración de soluciones de calcio se relaciona con la disminución de la incidencia del SHO, pero su efecto no es mayor al de la cabergolina (14).

\section{CONCLUSIÓN}

A medida que aumenta el uso de TRA a nivel mundial, aumenta la importancia del SHO como entidad clínica a incluir dentro de los diagnósticos diferenciales en mujeres que reciben estos tratamientos. Por lo anterior, resulta de relevancia la identificación de los síntomas iniciales que puede presentar este cuadro para orientar hacia un diagnóstico oportuno y un manejo adecuado de esta condición. Es por ello que la aplicación de medidas preventivas es fundamental ya que, aunque el $\mathrm{SHO}$ es una complicación rara, presenta implicaciones severas para estas pacientes. Ante esto, la educación y la buena comunicación médico-paciente se convierte en piedra angular que permitirá el acceso a una salud reproductiva cada vez más segura.

\section{ABREVIATURAS}

Síndrome de hiperestimulación ovárica (SHO); técnicas de reproducción asistida (TRA); gonadotropina coriónica humana (hCG); hormona folículo estimulante (FSH); fertilización in vitro (FIV); hormona luteinizante (LH); factor de crecimiento 
vascular endotelial (VEGF); síndrome de ovario poliquístico (SOP); hormona antimülleriana $\quad(\mathrm{AMH}) ; \quad$ hormona liberadora de gonadotropina $(\mathrm{GnRH})$.

\section{REFERENCIAS}

1. Humaidan P, Quartarolo J, Papanikolaou E. Preventing ovarian hyperstimulation syndrome: guidance for the clinician. Fertility and Sterility [en línea]. 2010 Jul [citado 26-marzo-2019]; 94(2): 389400. https://doi.org/10.1016/j.fertnstert.2010.03.028

2. Practice Committee of the American Society for Reproductive Medicine. Prevention and treatment of moderate and severe ovarian hyperstimulation syndrome: a guideline. Fertility and Sterility [en línea]. 2016 Dec [citado 25-marzo-2019]; 106(7): 1634-1647.https://doi.org/10.1016/j.fertnstert.2016.08.048

3. Olchowy A, Olchowy C, Łasecki M, Mazur R, Sierpowska M, Waligóra M et al. Ovarian Hyperstimulation Syndrome as a Growing Diagnostic Problem in Emergency Department Settings: A Case Report. The Journal of Emergency Medicine [en línea]. 2019 Feb [citado 26-marzo-2019]; 56(2): 217221. https://doi.org/10.1016/j.jemermed.2018.11.004

4. Nelson SM. Prevention and management of ovarian hyperstimulation syndrome. Thromb Res [en línea]. 2017 Mar [22-marzo-2019]; 151: 61-64. https://doi.org/10.1016/S0049-3848(17)30070-1

5. Elnory MA, Mahmoud Elmantwe AN. Comparison of cabergoline versus calcium infusion in ovarian hyperstimulation syndrome prevention: A randomized clinical trial. Middle East Fertility Society Journal [en línea]. 2018 Dec [citado 28-marzo-2019]; 23(4): 357-362.https://doi.org/10.1016/j.mefs.2018.05.001

6. Naredi N, Singh SK, Lele P, Nagraj N. Severe ovarian hyperstimulation syndrome: Can we eliminate it through a multipronged approach? Medical Journal Armed Forces India [en línea]. 2018 [citado 28-marzo2019];74(1):44-50. https://doi.org/10.1016/j.mjafi.2017.04.006

7. Abou Arkoub R, Wei Xiao C, Claman P, Clark EG. Acute Kidney Injury Due to Ovarian Hyperestimulation Syndrome. Am J Kidney Dis [en línea]. 2019 Mar [citado 28-marzo-2019]; 73(3): 416420. https://doi.org/10.1053/j.ajkd.2018.10.010

8. Tarlatzis BC, Bosdou JK, Kolibianakis EM. Ovarian Hyperstimulation Syndrome. Encyclopedia of Endocrine Diseases [en línea]. 2019 [citado 28-marzo-2019]; (2): 581-587.https://doi.org/10.1016/B978-012-801238-3.95856-2

9. Fatemi H, Garcia-Velasco J. Avoiding ovarian hyperstimulation syndrome with the use of gonadotropinreleasing hormone agonist trigger. Fertility and Sterility [en línea]. 2015 Apr [citado 30-marzo-2019]; 103(4): 870-873. https://doi.org/10.1016/j.fertnstert.2015.02.004

10. Navot D, Bergh PA, Laufer N. Ovarian hyperstimulation syndrome in novel reproductive technologies: prevention and treatment. Fertil Steril [en línea]. 1992 Ago [citado 28-marzo-2019]; 58: 249261. https://doi.org/10.1016/S0015-0282(16)55188-7

11. Shmorgun D, Claman P. No-268-The Diagnosis and Management of Ovarian Hyperstimulation Syndrome. $J$ Obstet Gynaecol Can [en línea]. 2017 Nov [citado 30-marzo-2019]; 39(11): 479486.https://doi.org/10.1016/j.jogc.2017.09.003

12. Balakumar V, Ramalingam M, Kay V. Ovarian hyperstimulation syndrome. Obstet Gynaecol Reprod Med [en línea]. 2017 Dec [citado 30-marzo-2019]; 27(12): 357-362.https://doi.org/10.1016/j.ogrm.2017.10.002

13. Bellapu S, Guttman J. Use of Point-of-Care Ultrasound for the Diagnosis of Ovarian Hyperstimulation Syndrome. J Emerg Med [en línea]. 2017 Apr [citado 31-marzo-2019]; 52(4): 101104. https://doi.org/10.1016/j.jemermed.2016.11.026

14. Namavar Jahromi B, Parsanezhad ME, Shomali Z, Bakhshai P, Alborzi M, Vaziri NM, et al. Ovarian Hyperstimulation Syndrome: A Narrative Review of Its Pathophysiology, Risk Factors, Prevention, Classification, and Management. Iran J Med Sci [en línea]. 2018 May [citado 22-marzo-2019]; 43(3): 248260. Disponible en: https://europepmc.org/articles/PMC5993897 
15. Sociedad Española de Ginecología y Obstetricia. Prevención y manejo del síndrome de hiperestimulación ovárica (2017). Prog Obstet Ginecol [en línea]. 2018 [citado 01-abril-2019]; 61 (4): 410-416. Disponible en: https://sego.es/documentos/progresos/v61-2018/n4/19 GAP\%2023J.-

\%20Prevenci\%C3\%B3n\%20y\%20manejo\%20del\%20S\%C3\%ADndrome\%20de\%20hiperestimulaci\%C3 \%B3n\%20ov\%C3\%A1rica\%20(2017).pdf

16. Lele $\mathrm{P}$, Tangri M. A case of ovarian hyperstimulation syndrome with spontaneous hemoperitoneum. International Journal of Reproduction, Contraception, Obstetrics and Gynecology [en línea]. 2017 Dic [citado 23-abril-2019]; 6(2): 766-768. http://dx.doi.org/10.18203/2320-1770.ijrcog20170421

17. Dincgez Cakmak B, Ozgen G, Dundar B, Ketenci Gencer F. Management of Bilateral Adnexal Torsion in a Case of Ovarian Hyperstimulation Syndrome. European Archives of Medical Research [en línea]. 2018 Set [citado 23-abril-2019]; $34(3)$ : 196-199. Disponible en:https://www.journalagent.com/eamr/pdfs/EAMR 343196 199.pdf

18. Smith V, Osianlis T, Vollenhoven B. Prevention of Ovarian Hyperstimulation Syndrome: A Review. Obstetrics and Gynecology International [en línea]. 2015 May [citado 01-abril-2019]; 2015: article ID 514159. http://dx.doi.org/10.1155/2015/514159

19. Fischer D, Reisenbüchler C, Rösner S, Haussmann J, Wimberger P, Goeckenjan M. Avoiding OHSS: Controlled Ovarian Low-Dose Stimulation in Women with PCOS. Geburtshilfe Frauenheilkd [en línea]. 2016 Jun [citado 03-abril-2019]; 76(6): 718-726. https://doi.org/10.1055/s-0042-100206

20. Nastri CO, Teixeira DM, Moroni RM, Leitão VMS, Martins WP. Ovarian hyperstimulation syndrome: pathophysiology, staging, prediction and prevention. Ultrasound Obstet Gynecol [en línea]. 2015 Mar [citado 03-abril-2019]; 45(4): 377-393. https://doi.org/10.1002/uog.14684 